\title{
La Educación desde una perspectiva de la complejidad
}

\author{
Margarita Ramírez Ramírez \\ maguiram@uabc.edu.mx \\ ORCID 0000-0002-4252-4289 \\ Facultad de Contaduría y Administración, \\ Universidad Autónoma de Baja California
}

\author{
Esperanza Manrique Rojas \\ emanrique@uabc.edu.mx \\ ORCID 0000-0003-1928-9353 \\ Facultad de Contaduría y Administración, \\ Universidad Autónoma de Baja California
}

\author{
Ismael Plascencia López \\ ismael@uabc.edu.mx \\ ORCID 0000-003-2860-1417 \\ Facultad de Contaduría y Administración, \\ Universidad Autónoma de Baja California
}

Recepción: 17/09/2021

Aceptación: 27/10/2021

\section{Resumen}

En el presente artículo se realiza una reflexión sobre el enfoque de la complejidad en modelos educativos, los cuales cada día se alejan de modelos reduccionistas ante las carencias que este enfoque de la ciencia presenta. Se analizan características de un sistema complejo como la emergencia, la autoorganización, que caracterizan a los sistemas complejos. Se destaca la complejidad y sus características, como factores indispensables en un modelo educativo que atienda a las necesidades de educación requeridas en un entorno globalizado, cambiante y complejo que presenta desafíos de manera constante.

El presente artículo parte del análisis del concepto de reduccionismo, que ha sido un método exitoso para alcanzar avances significativos en la ciencia; sin embargo, ante las condiciones actuales, las necesidades de un mundo globalizado, es necesario implementar nuevos modelos educativos que ofrezcan integración de componentes y análisis holísticos de problemáticas para ser atendidas y resueltas.

Se analizan los principios de complejidad y sistemas complejos, para realizar una descripción de un modelo educativo con una perspectiva de la complejidad y paradigmas de complejidad y educación.

Palabras clave: Complejidad, Sistemas complejos, educación. 


\section{Abstract}

This article presents a reflection on the complexity approach in educational models, which every day move away from reductionist models due to the deficiencies that this approach to science presents. Characteristics of a complex system such as emergence, self-organization, that characterize complex systems are analyzed. Complexity and its characteristics are highlighted as indispensable factors in an educational model that meets the educational needs required in a globalized, changing and complex environment that constantly presents challenges.

This article starts from the analysis of the concept of reductionism, which has been a successful method to achieve significant advances in science; However, given the current conditions, the needs of a globalized world, it is necessary to implement new educational models that offer integration of components and holistic analysis of problems to be addressed and resolved.

The principles of complexity and complex systems are analyzed to make a description of an educational model with a perspective of complexity and paradigms of complexity and education.

Keywords: Complexity, Complex systems, education.

\section{Introducción}

Se suele afirmar que con la revolución tecnológica y la globalización el mundo se ha vuelto más complejo. En realidad, el mundo desde siempre ha sido complejo, sin embargo, en los últimos años se reconoce la necesidad de contar con áreas de la ciencia que nos permitan explicar y resolver problemas en los que concurren una gran cantidad de componentes, interacciones, causas y efectos emergentes no proporcionales y transversales, en muchas ocasiones no parametrizables y por tanto difícilmente de ser pronosticables. Las ciencias de la complejidad se han aplicado en la investigación científica, asi como en el desarrollo tecnológico, pero sobre todo son una alternativa para abordar y proponer soluciones a los problemas sociales que afectan a nuestro entorno. Esto es, los planes, programas, proyectos deben ser orientados a atender problemas complejos de desarroIlo social, en sus distintos ámbitos: económico, social, cultural, educativo, de salud, entre otros. Para abordar de manera efectiva las problemáticas de la comunidad, los programas públicos requieren ser diseñados bajo un enfoque de sistemas complejos. Programas que comúnmente son interdisciplinarios, transdisciplinarios e interinstitucionales, que involucran condiciones complejas de muy diversa naturaleza, que se entrelazan y derivan en fenómenos emergentes más agudos.

Para diseñar estos programas se requiere la Inte- gración de equipos de investigación y académicos que actúen con una visión sistémica, crítica, creativa y humanista. Se requieren propuestas bajo un esquema de redes de participación colaborativa multidisciplinaria entre sociedad y gobierno. Se deben abordar utilizando herramientas propias para el estudio de sistemas complejos; Sin embargo, no es un cambio sencillo, requiere proyectos y aplicaciones concretas, así como programas específicos y la inversión en infraestructura, recursos humanos, científicos y tecnológicos.

\section{Reduccionismo}

En los avances de la ciencia se han dividido y simplificado los fenómenos en sus partes para poder estudiarlos de manera más concreta, a este enfoque se le llama reduccionismo.

El reduccionismo es un enfoque filosófico, en el cual la reducción es suficiente y necesaria para resolver distintos problemas de diferentes áreas de conocimiento.

Este método se ha utilizado de manera exitosa en áreas como la ingeniería, en la que a partir de la construcción de partes independientes se construyen sistemas con funcionalidad esperada. Es el reduccionismo un aliado en la creación de componentes especializados para el diseño de computadoras y dispositivos electrónicos. A pesar de las ventajas que ofrece en el área de ingeniería computacional el reduccionismo, es necesario para el avance y tener interacciones que cambian la funcionalidad. Se reconoce que el reduccionismo ha sido necesario para el desarrollo de la ciencia; Sin embargo, una vez que se han logrado avances en la ciencia ha sido necesario incorporar conceptos de sistemas, y sus interrelaciones y por lo tanto observar a los sistemas complejos.

A pesar de ser muy útil, el reduccionismo tiene sus límites, cuando se analiza un sistema y se reconocen las interacciones entre sus componentes, el reduccionismo se vuelve inadecuado, ya que al separar y simplificar se pierde información y no se consideran los resultados de las interacciones (Gershenson,2015).

Por otra parte, el reduccionismo consiste en tomar un aspecto de la realidad y en considerarlo como lo característico o lo más importante para el ser humano por lo que se puede llegar a una realidad de lo que se percibe o deduce (Daros, 1991). Sin embargo, en el área de medicina, el reduccionismo ha permitido grandes avances, con estos avances en diferentes especialidades; sin embargo, el reduccionismo es limitado para atender enfermedades complejas, ya que pueden tener causas múltiples, por lo que no es posible tener una sola cura, es necesario atender las diversas causas con diferentes tratamientos. Por lo tanto, en la medicina cada vez más se realizan la atención de enfermedades desde la perspectiva de un sistema complejo. 
El reduccionismo se da al tomar posturas restrictivas en relación a la creación y construcción del conocimiento, se da al ser considerado que el todo, no es más que la suma de las partes que lo conforman (Pérez-Tamayo, 2011).

La educación no es la excepción, es necesario visualizarlo con enfoques globales y no solo desde aspectos limitados o específicos.

\section{Reduccionismo en educación}

Es posible considerar el reduccionismo en el ambito académico, expresado a partir de una desagregación conceptual de los contenidos de una disciplina abordada en los distintos niveles educativos (Reyes-Rodríguez, et. al, 2017).

En el área de educación el reduccionismo define que hay una técnica adecuada para aplicar el método enseñanza-aprendizaje; el reduccionismo en el área de educación limita a las estrategias metodológicas, el caso del aprendizaje por competencias es una metodología reduccionista. En la educación la mejor alternativa es integrar estrategias, técnicas, y considerar factores físicos, sociales y culturales, reconocer la diversidad de contextos, variables, actores, hacer el uso de múltiples metodologías, que ofrezcan soluciones efectivas en los procesos enseñanza-aprendizaje. Esta interacción e integración de componentes abre la posibilidad de considerar a la educación como un sistema complejo.

Para analizar las condiciones de un modelo educativo basado en la ciencia de la complejidad, analizamos los conceptos y características de complejidad y de un sistema complejo.

\section{Complejidad}

La teoría de la complejidad se ha desarrollado en ciencias y humanidades. La primera se conoce como ciencias de la complejidad, mientras que la segunda se denomina generalmente pensamiento complejo. Las ciencias de la complejidad utilizan lenguajes formales para modelar y simular sistemas complejos. Mientras que el pensamiento complejo construye sus teorizaciones en lenguaje natural (Rodríguez Zoya, L. G. (2020)).

La complejidad, no es un tema único de las sociedades humanas, sino de la naturaleza misma. Podemos considerar que la sociedad es una manifestación de una organización única que permanece ligada a un entorno vivo que integra la naturaleza, la complejidad integra a una realidad perceptible por la conciencia humana, este término puede describir a la realidad e integrar una variedad de elementos y factores, los cuales a su vez están integrados por fenómenos complejos.

Pensar en complejidad es considerar problemas sociales y fenómenos de la naturaleza integrados por componentes con conexiones diversas, con ciclos impredecibles en el que cada proceso o suceso puede generar situaciones diversas, en el que causas iguales generan resultados diferentes y existen relaciones multidireccionales.

La complejidad se fortalece del desarrollo de la investigación de distintas disciplinas y determina el fortalecimiento de las disciplinas (Nicolescu, sf). Complejidad permite analizar, considerar determinados aspectos de la naturaleza, de la sociedad y del pensamiento, que pueden presentar características y comportamientos que puede clasificarlos como sistemas complejos. La complejidad considera la concepción del universo como un ente, el cual es más que la suma de las partes que lo componen y que es fortalecido por sus componentes y la interacción y relación que se da entre sus partes, son capaces de generar nuevas formas. Este paradigma científico busca conexiones entre investigadores, que trabajan en temas de ciencia de la frontera de diferentes disciplinas (Hayles, 1998).

En las ciencias, es posible considerar complejidad como una forma de pensamiento, como un comportamiento colectivo que lo integran elementos que pueden ser humanos, físicos, situacionales, que conforman a un grupo, átomo, o moléculas que conforman a un objeto, neuronas, bits de computadora, entre otros, las posibles interacciones entre los componentes y los principios de causa efecto que puede tener una relación no lineal entre los elementos, como el efecto mariposa, y propiedades emergentes de los sistemas, entre los cuales cada sistema es mayor a la. integración de sus partes.

\section{Sistema Complejo}

Para iniciar nuestra reflexión, se analiza a que se refiere el término de sistema complejo, podemos partir del concepto general de un sistema complejo, los cuales están formados por diferentes subsistemas o elementos, que interactúan entre sí de manera dinámica, se modifican con el tiempo, interactúan de manera no lineal, debido a que no hay una relación directa entre las causas y efectos. De acuerdo a Lara (2016) un sistema complejo se caracteriza porque son: Agentes biológicos, psíquicos y sociales, los cuales tienen intenciones u objetivos diversos, dinámicos, con prioridades diferentes y pueden ser contradictorios, de igual manera son agentes que al actuar modifican la realidad y son modificados por ésta, que tienen propiedades emergentes que surgen a partir de las interacciones entre ellos y que presentan procesos de auto-organización, de igual modo presentan estados de caos y horizontes de predictibilidad, integran agentes y colectivos que presentan percepción, homeostasis, acción, adaptación y resiliencia. 
Analizaremos estos conceptos de complejidad en un entorno educativo, no antes de realizar una revisión a la conceptualización de diversos especialistas de ciencias de la complejidad de lo que es un sistema complejo. En el análisis de diversas conceptualizaciones de sistemas complejos encontramos similitudes como la interrelación entre sus componentes, interrelaciones no lineales y dinámicas y de estas interacciones surgen nuevas propiedades, las cuales pueden identificar como emergentes.

Mitchell (2009), menciona: Un sistema complejo es un sistema integrado por componentes interrelacionados que no cuentan con un control central, ni ccuentan con reglas de operación entre los componentes las cuales originan un entorno colectivo, puede ser el procesamiento de información y la adaptación de sus elementos través del aprendizaje y el avance de estos (Mitchell, 2009). Por su parte Luhmann (2012), expone que un sistema complejo, a partir de la operación, emerge cuando un proceso inicia y es posible la conexión con otros procesos de un mismo tipo y continuar produciendo operaciones del mismo tipo. Lara, expone en su obra que un sistema complejo es una estructura jerárquica de entidades compuestas por subsistemas interrelacionados a varios niveles. Cada uno de estos subsistemas está compuesto por subsistemas interrelacionados a nivel inferior, y así sucesivamente. Una constante que existe es el que las interrelaciones entre todos los niveles son dinámicas y no lineales, en algunos casos circulares, llamadas retroalimentaciones (Lara-Rosano,2012).

Para Martínez (2013), un sistema complejo es una organización o grupo, que se construye a. partir de la interacción de elementos primarios. En estos sistemas, los elementos que lo componen y la interacción que se da entre ellos conducen a comportamientos que en su mayoría no son fáciles de predecir desde un conocimiento base, el efecto de este comportamiento es conocido como emergente (Martínez, 2013).

Por su parte (Álvarez-Buylla \& Frank,2013) expone que un sistema complejo está comformado por elementos interrelacionados, entrelazados, en el que las interacciones propician comportamientos que generan información adicional, que comunmente estan ocultas para el observador. Como resultado de la interacción de los elementos, se generan propiedades reconocidas como emergentes, que no es posible explicarlas a partir de propiedades de los elementos aislados.

En coincidencia con (Álvarez-Buylla \& Frank, 2013) (Gershenson, 2007), menciona que el comportamiento de cualquier sistema, es difícil de deducir a partir de solo el comportamiento de las partes que lo componen. Para (Morín,1994), complejidad no puede reducirse a una idea simple, ni únicamente al término de complejidad.
Una de las características de un sistema complejo, es que es un sistema abierto, que interactúa con el ambiente y que es capaz de establecer relaciones de cooperación o competencia con otros sistemas. Las relaciones que se establecen en un sistema complejo, no solo son interacciones entre sus elementos o participantes, sino también las relaciones que se dan de este sistema con otros sistemas en el entorno. (De Jesús Lara Lozano et al., n.d.)

Es importante concluír que los comportamientos colectivos son impredecibles y no lineales, que la característica más común de estudio en un sistemas complejos es que cuentan con propiedades que pueden reconocerse como emergentes (Boccara, 2004), esto gracias a la autoorganización que se genera entre sus componentes y que estas propiedades emergentes no pueden ser determinadas por modelos analíticos (Byrne, 1998).

\section{La Educación desde una perspectiva de la com- plejidad}

Considerando las condiciones emergentes de la complejidad, la educación es un tema que debe ser analizado desde esta perspectiva. En este momento la educación a partir de los principios de complejidad es la concepción de un proceso de educación hacia la conciencia de un mundo en el que es posible considerar diferentes opiniones, encontrar contradicciones y aceptar a la educación como un proceso global integrador, con diversidad de. El pensamiento complejo propicia la adquisición de conocimiento a partir del avance en el día a día y la interrelacción de los elementos que conforman el proceso. Proceso en el que el aprender trasciende disciplinas, en el que es necesario considerar macroconceptos. Que pueden traspasar áreas de conocimiento a otra, en la que es posible que emerjan nuevas formas de lograr el aprendizaje, con una práctica más sensible, propiciando aprendizaje autónomo, con herramientas y habilidades para integrar el entorno como parte de los constructos del saber. La educación desde una perspectiva de complejidad debe ser pertinente con una visión integradora, desde la comprensión y la construcción de un fenómeno educativo humano, conformado por varias dimensiones, que sea integrador, intercultural, que se de a través de la transdisciplinariedad, que sea posible reconocer el error, que acepte la incertidumbre y la diversidad (De Jesús, et. al,2007)

Si nos remontamos a los principios de la humanidad encontramos a la educación como una de las bases fundamentales, sobre los cuales las comunidades establecen procesos transformadores en sus hábitos, en sus costumbres, en sus valores y en la adquisición de sus conocimientos. La educación ha sido reconocida como la forma tradicional de la transmisión de conocimientos; Sin embargo nuevas perspectivas del proceso educati- 
vo muestran a este como el proceso de construcción de conocimiento a través de experiencias captadas por nuestros sentidos, de acuerdo a (López,2013), Maturana, señala que el conocer es el hacer del que conoce, y esto está basado en la misma manera de su ser vivo, en su organización (López et al., 2003) .Es importante considerar el proceso de autoorganización y reconocer a la educación como un proceso, perfectible, en el que no solo participan los estudiantes y docentes, es un proceso en el cual se involucran alumnos, profesores, autoridades administrativas de las instituciones, investigadores, familiares, políticos, tomadores de decisiones, etc. La educación como poiésis (creación), considerando el contexto en el proceso educativo y todos los involucrados.

\section{Paradigma de la complejidad y la educación}

A partir de las nuevas concepciones del proceso enseñanza-aprendizaje en el que el conocimiento se construye a partir de nuevas consideraciones y formas de interacción de componentes, en el que el conocimiento es una realidad en proceso, en la que se considera al sujeto junto a sus sentimientos, actitudes, aptitudes y esto hace un conjunto de seres con características y visiones heterogéneas, surge el concepto de pensamiento complejo.

Desde una epistemología de la complejidad, la educación debe ser abordada a partir de un enfoque sistémico, no lineal, como un pensamiento que avanza en conocimiento, y se desarrolla a partir de los integrantes y condiciones del proceso.

Morín presenta una propuesta educativa en cuanto a complejidad, considerando un conocimiento multidimensional, que es capaz de abordar las problemáticas integrando diferentes disciplinas, aceptando a la incertidumbre, y a la diversidad como elementos intrínsecos de los procesos educativos. (Morín, 1993), expone:

"Los elementos de todo sistema se organizan según una finalidad. La educación es un sistema y como tal plantea el requerimiento de realizar una reflexión sobre el funcionamiento real de sus componentes en interacción con el contexto, teniendo en cuenta su interacción y evolución a largo plazo"

Conforme pasa el tiempo, surgen problemas que propician a la consideración de pensar en nuevas formas de enseñar, esto es la necesidad de enseñar desde un enfoque interdisciplinario, multidisciplinario o transdisciplinario, que permita atender a un problema desde diferentes perspectivas, y poder realizar una solución adecuada, y que se mantenga en vigencia por periodos amplios de tiempo, de igual forma Estrada García (2017) sugiere en relación a los modelos educativos se requiere fortalecer la autonomía, el emprendimiento, la independencia, el liderazgo, así como la capacidad de los estudiantes de generar su propio conocimiento; que el estudiante sea capaz de autoformarse, de aprender a aprender, a través de una metodología interdisciplinaria. En relación a los docentes, Collado R et al (2018) consideran que hay dimensiones que pueden ser formativas, que los docentes del siglo XXI, deben considerar una educación transdisciplinaria, que permita abordar problemáticas desde diferentes disciplinas, por lo tanto reconocer la multidimensionalidad y el dinamismo que se da en los fenómenos que interactúan en la formación humana.

En adición a lo anterior, se debe avanzar, en ir más allá de la reducción o disyunción de opciones y pensar en conjunto, con el propósito de cambiar lo fragmentado desde una perspectiva transdisciplinar. Es necesario considerar que la complejidad no busca un conocimiento general, por el contrario, ofrece alternativas para detectar las conexiones, las articulaciones que existen en un sistema, sea cual sea este, aspectos de naturaleza humana, sociedad, cultura, etc.

Por su parte (Flores JH, 2015), expone que la educación en referencia de las diferentes disciplinas requiere de un carácter de diálogo, de comunicación, que sea capaz de salir del área de especialización para confrontarse con otras áreas y que se integren en la verdad. En esta visión epistémica, el conocimiento no es solo la representación de una realidad definida anteriormente, es más bien un proceso que se construye de manera significativa, y con una relación con las áreas relacionadas. El pensamiento complejo permite unir, integrar, construir y vincular conocimientos, los cuales comúnmente están en constante cambio. (Morín, 1994).

\section{Conclusiones}

En esta era de la cuarta revolución industrial y globalización en la que el proceso de innovación humana y desarrollo tecnológico ha logrado avances inimaginados, en los que las áreas de conocimiento se han integrado, es sin duda una época con un contexto complejo que requiere realicemos un análisis y diseño de modelos educativos que reconozcan nuevas formas de aprender y que nos apoye en el enfrentamiento de la educación desde un mundo complejo, globalizado, intercomunicado y autoorganizado. Lo cual no es suficiente con el diseño de planes y programas de estudio que ofrezcan el contenido de las nuevas tecnologías y áreas de la ciencia requerida, sino que se promueva un cambio en la integración de nuestros pensamientos y construcciones mentales. Un modelo educativo que no separa a las ciencias exactas y duras de las ciencias blandas, por el contrario que sea capaz de integrar áreas y disciplinas en la concepción y solución de problemáticas 
actuales, que se prepare para ser parte de un sistema, que se combine lo cuantitativo con lo cualitativo y que se promueva procesos de cambio e integración de áreas y autoorganización. Que sean considerados elementos que lo conforman como: docentes, estudiantes, instituciones educativas, planes y programas de estudio, instituciones gubernamentales, estructuras económicas, políticas, entornos laborales y sobre todo la interacción entre cada uno de estos componentes.

\section{Referencias Bibliográficas}

Álvarez-Buylla, E. y Frank H. A. (2013). El Centro de ciencias de la complejidad de la UNAM: Piedra de roseta para la ciencia en México, Interdisciplina,I (1), 171180.

Byrne, D.S. (1998). Complexity Theory in the Social Sciences: An Introduction. Routledge

Collado R. et al. (2018) L F. Educación transdisciplinar: formando en competencias para el buen vivir. Ensaio: Avaliação e Políticas Públicas em Educação, Rio de Janeiro, v. 26, n. 100 , p. 619-644, Recuperado de: https://doi.org/10.1590/s0104-40362018002601487

De Jesús, María Inés, Andrade, Raiza, Martínez, Don Rodrigo, Méndez, Raizabel Re-pensando la Educación desde la Complejidad. POLIS, Revista Latinoamericana [en linea]. 2007, 6(16), 1-13[fecha de Consulta 7 de noviembre de 2021]. ISSN: 0717-6554. Recuperado de: https://www.redalyc.org/articulo. oa?id=30501602.

Daros WR, (1991), Aprendizaje y educación en el contexto del humanismo., Revista española pedagógica [en línea] ISSN 2174-0909

recuperado de: https://revistadepedagogia.org/ wp-content/uploads/2018/03/4-Aprendizaje-y-Educaci\%C3\%B3n-en-el-Contexto-del-Humanismo.pdf

Estrada Garcia T, A. (2017); Respuesta a la matriz productiva desde la educación en ciencia, tecnología y sociedad (ECTS) y la metodología sistémica. Revista Arbitrada del Centro de Investigación y Estudios Gerenciales, [s. I.], v. 5, n. 27, p. 236-249., sept. 2017. Recuperado de: https://goo.gl/aid1Qd.

F. Lara. (2016). Las ciencias de la complejidad en la solución de nuestros problemas sociales. Sistemas, cibernética e informática, Vol. 36, 50. Recuperado: http://www.iiis.org/CDs2016/CD2016Spring/papers/ CB076IQ.pdf

Flores JH (2015). Complejidad y educación.Repositorio Digital de la Ciencia y cultura de El Salvador, REDICCES,Editorial Universidad Don Bosco.

Gershenson, C. (2007). Design and control of Self-organiz- ing Systems. CopIt ArXives.

Gershenson (2015), Reduccionismo: sus ventajas y sus límites, Investigación y Ciencia https://www.investigacionyciencia.es/blogs/fisica-y-quimica/34/posts/reduccionismo-sus-ventajas-y-sus-Imites-13230.

Hayles, K (1998). La evolución del caos. El orden dentro del desorden en las ciencias contemporáneas. BarceIona. Gedisa

Lara-Rosano, F. (2012). Teoría, métodos y modelos de la complejidad social I. Seminario de Investigación. CCADET.

López Melero, M.; Maturana, H. y et al. (2003), Conversando con Maturana de educación. Málaga, España: Ediciones Aljibe.

Luhmann, N. (2012). Introduction to Systems Theory.Polity Press.

Martínez, G., Adamatzky, A. \& Alonso-Sanz, R. (2013). Designing Complex Dynamics in Cellular Automata with Memory, International Journal of Bifurcation and Chaos 23(10), 1330035 -131.Recuperado de http://www.worldscientific.com/doi/abs/10.1142/ S0218127413300358.

Mitchell, M. (2009). Complexity: A guided tour of our.USA: Oxford University Press.

Morin, E. (1993). El método I. Naturaleza de la naturaleza. Madrid. Cátedra.

Morin, E. (1994). Introducción al pensamiento complejo. Barcelona, SPAIN: Gedisa.

N. Boccara "Modelling Complex Systems ", (Springer-Verlag, 2004).

Nicolescu, B. (s.f.). La transdisciplinariedad. Manifiesto, Ediciones Du Rocher.

Pérez-Tamayo, R. Acerca de Minerva. 3. ed. Ciudad de México: Fondo de Cultura Económica, 2011.206 p.

Reyes-Rodríguez, Rondero-Guerrero,Acosta-Hernández, Campos-Nava,Torres-Rodríguez (2017), Reduccionismo Didáctico y creencias de profesores acerca del teorema de Pitágoras, UNESP, https://doi. org/10.1590/1980-4415v31n59a06

Rodríguez Zoya, L. G. (2020). Ciencia, política y problemas complejos. Revista Ciencias De La Complejidad, 1(1), 37-49. https://doi.org/10.48168/cc012020-003

Saldarriga, Aguirre. (2014). Sistemas de innovación como sistemas complejos. Medellín: InstitutoTecnológico Metropolitano. Recuperado de: https://books.google.com. $\mathrm{mx} /$ books?id=Vfc-DwAAQBAJ\&pg=PA73\&dq=Sistemas+complejos+adaptativos\&hl=es\&sa $=X \& v e d=0 a-$ hUKEwjVvcrf3JDeAhWSDuwKHSrMCsUQ6AEIM$\mathrm{TAC} \# \mathrm{v}=$ onepage $\& \mathrm{q}=$ Sistemas $\% 20$ complejos $\% 20$ adaptativos $\& f=$ false. 